\title{
Sistema de Información Soportado en Recuperación XML para Pequeñas y Medianas Empresas (PYME) de Cartagena de Indias, Colombia
}

\author{
Amaury Cabarcas, Plinio Puello, Raúl J. Martelo \\ Universidad de Cartagena, Facultad de Ingeniería, Grupo de Investigación en Tecnologías de las \\ Comunicaciones e Informática, GIMATICA, Avenida del Consulado, Calle 30, No. 48 - 152, Cartagena- \\ Colombia. (e-mail: acabarcasa@unicartagena.edu.co, ppuellom@unicartagena.edu.co, \\ rmartelog1@unicartagena.edu.co)
}

Recibido Jul. 25, 2014; Aceptado Oct. 13, 2014; Versión final recibida Dic. 12, 2014

\begin{abstract}
Resumen
El objetivo de este artículo es presentar un sistema de información soportado en recuperación XML como una herramienta para el apoyo en la gestión de órdenes de trabajo en una PYME de Cartagena para generar información que apoye el proceso y en la toma de decisiones. Se utilizó como metodología de desarrollo una estructura de proceso general de la ingeniería de software. Se determinó la utilidad del software mediante el diseño, construcción e implementación en las PYME, validando su uso a través de una prueba de usabilidad. Lo anterior permitió medir el grado de aceptación de los usuarios finales, obteniendo como resultados ahorro en costos en la gestión del archivo histórico y apoyo en la toma de decisiones. Basado en los resultados obtenidos, se concluye que la gestión de órdenes de trabajo se ve optimizada al implantar el sistema de información.
\end{abstract}

\section{Information System Supported in XML Retrieval for Small and Medium Size Enterprises (SME) from Cartagena de Indias, Colombia.}

\begin{abstract}
This paper presents the development of an information system using XML retrieval, as a tool to support the management of work orders in small and medium size enterprises (SME) from Cartagena to generate information to support decision making in the process outlined. The methodology used was the development of a structure of the general process of software engineering. The usefulness of the software was determined by the design, construction and implementation in the SME, validating its use through a usability test. This allowed measuring the level of acceptance of end users, thus saving costs in managing archived files and facilitating decision making. Based on the results it was possible to conclude that the process of work order management was improved by implementing the information system
\end{abstract}

Keywords: information retrieval, XML retrieval, information systems, SME 


\section{INTRODUCCIÓN}

La capacidad de innovar representa hoy, el principal determinante del incremento de la productividad y la competitividad en las empresas y, por ende, de los países y regiones, siendo los resultados de esa capacidad la principal fuente de ventajas competitivas dinámicas (Rodríguez, et al., 2011). Dentro de los obstáculos que las pymes tienen para su desarrollo en Colombia, se han podido identificar: las dificultades en la identificación y acceso a la tecnología adecuada, la formalización y absorción de nuevas tecnologías, entre otros (Hernández, et al., 2014). Es por eso que el uso y apropiación por las organizaciones de las herramientas tecnológicas dentro de las que se incluyen el software y los sistemas de información, ha sido una de las formas en que las éstas han buscado generar ventajas competitivas. Ejemplos de la afirmación anterior los podemos encontrar en diversos sectores (Puello, et al., 2013; Carrillo y Franky, 2014). Es así que a nivel internacional en distintos estudios sobre competitividad realizados (United Nation Conference on trade and Development, 2012), han demostrado que la implementación de herramientas tecnológicas como el software y la masiva utilización de sus servicios en las empresas, pueden convertirse en una herramienta para volverse más dinámicos y estar a la altura de las grandes industrias o economías a nivel mundial.

Para la implementación y el uso de estas herramientas tecnológicas se debe tener en cuenta el tipo de empresa y cada una de las necesidades individuales, en este artículo se aborda el caso de la empresa "La casa del embobinador", esta empresa cartagenera ofrece servicios de mantenimiento y reparación de máquinas eléctricas, en este caso bobinas eléctricas. En el funcionamiento normal de la empresa, estos procesos se llevan a cabo de manera manual, utilizando un formato impreso llamado orden interna de trabajo (OIT) el cual es diligenciado por el operario encargado de la reparación y luego digitados en hojas de cálculo y los registros manuales de las OIT son archivados. La empresa cuenta con un archivo histórico extenso y se ha visto en la necesidad de tomar como política la eliminación de parte de los documentos cada dos años, en promedio se procesan entre 180 y 200 OIT mensuales, alrededor de 2400 anuales lo que hace que la empresa que tiene más de 20 años no pueda manejar un registro de alrededor 60.000 documentos archivados. Adicionalmente a las OIT se le asocian diseños gráficos estándares o a particulares que muestran la estructura de embobinado y algunos documentos de interacción con el cliente y con los técnicos de reparación, el proceso de búsqueda y extracción de información para la toma de decisiones, demanda una gran cantidad de tiempo y recursos humanos. Para alcanzar solucionar el problema mencionado arriba se desarrolló un sistema de información para la gestión de OIT soportado en recuperación XML, con el fin de apoyar la toma de decisiones y la búsqueda de ý extracción de información del registro histórico.

La mayoría de los software, son desarrollados con el fin de manipular grandes cantidades de información, el objeto principal es poder encontrar esa información cuando a cualquier persona le surja una necesidad que pueda ser cubierta con un conjunto de esos datos previamente almacenados. El diseño y desarrollo de bases de datos relacionales, en un principio, pareció el apoyo perfecto para estos desarrollos (Martínez, 2004). Recientemente el concepto de búsqueda y recuperación de información, en inglés information retrieval (IR) ha venido siendo aplicado en diversos sistemas (Manning, Raghavan, y Schutze, 2009). Los sistemas que usan IR han sido desarrollados e implementados en varios proyectos a nivel internacional (Vélez, et al. 2004) desarrollaron un sistema intermedio para manejar datos terrestres que facilitaran la colaboración entre un conjunto de proveedores, quienes desean compartir una imagen o un conjunto de datos. Igualmente (Pokorny, 2004) muestra la importancia de los servicios web de primera información a partir de las técnicas, y algoritmos de la IR tradicional. Este trabajo presenta una visión general de las arquitecturas de motores de búsqueda y técnicas en el contexto de IR. También se encuentran trabajo relacionados como el de (Hassler, 2005) en el cual ofrece y describe bibliotecas de código abierto a los desarrolladores usando técnicas de IR en la construcción de escritorios personalizados y funciones de búsqueda empresarial. En la actualidad la búsqueda y recuperación de información esta implementada en la mayoría de los software existentes, en especial en aquellos en que el volumen de informaciones significativo, son unas técnicas que a medida que el tiempo transcurra y consigo los constantes cambios de la tecnología, llevara a que estos métodos se adapten a las exigencias del medio, con el objetivo primordial de ser eficaz y eficiente en todo momento.

Con el fin de validar el potencial uso del software se realizó una prueba de usabilidad; con los empleados de la empresa. Como principal resultado se encontró que el enfoque propuesto presenta ventajas que van desde el ahorro en costos en el manejo del archivo histórico hasta el apoyo en la gestión de las OIT, pasando por la aplicación de recuperación XML en un sistema de información; lo que permitió incluir un aspecto novedoso en el sistema de información el cual es útil y efectivo para el fin propuesto. Recuperación XML, o XML Retrieval (Luk et al, 2002; Manning et al., 2009), es una de las técnicas de recuperación de información basada en el contenido de los documentos estructurados usando XML (eXtensible Markup Language) (Bray et al, 1998). Esta técnica es utilizada para calcular la relevancia de la información estructurada en documentos XML en los sistemas de recuperación de información (Winter y Drobnik, 2007). 


\section{FUNDAMENTOS TEÓRICOS}

La importancia de las micro, pequeñas y medianas empresas (Mipymes) dentro del tejido socioeconómico de cualquier país es indiscutible, en variables como número de establecimientos, empleo, producción, distribución del ingreso, entre otros indicadores (BID, 2005; Malhotra et al, 2007; Zevallos, 2007; Galindo, 2005); de esto se deriva un interés creciente por investigar la situación de estos negocios y por generar e implementar programas para la competitividad de la misma, buscando su supervivencia ante el fuerte aumento de los niveles de competencia global. En el caso colombiano, la definición de la Pyme como estrato productivo está contemplada en la legislación del país (Congreso de la Republica de Colombia, 2000), en la cual se establecen los parámetros de clasificación de las pymes dentro de los que se destacan: número de trabajadores y activos totales. Esta categorización jurídica hace referencia a las Mipymes, como unidades de explotación económica, realizadas por persona natural o jurídica, en actividades empresariales, agropecuarias, industriales, comerciales o de servicios, rural o urbana. De acuerdo al artículo $2^{\circ}$ de la Ley 590 de 2000: serán clasificadas como Mediana, aquellas empresas que tienen una planta de personal entre 51 y 200 trabajadores y activos totales por valor entre 100.000 a 610.000 unidades de valor tributario (UVT). Pequeña empresa, con una planta de personal entre 11 y 50 trabajadores y Activos Totales por valor entre 501 y menos de 5.000 salarios mínimos mensuales legales vigentes (SMMLV). Microempresa, con una planta de personal no superior a los 10 trabajadores y unos Activos Totales inferior a 500 SMMLV, excluida la vivienda. Partiendo de estas clasificaciones, se estima que las Mipymes son relevantes, ya que son la base de la economía, en 2009 representaban el 96.4\% del parque empresarial nacional, las cuales generaron el $80 \%$ del empleo total (Plata, 2009).

Los sistemas de información son un conjunto de elementos interrelacionados con el propósito de prestar atención a las demandas de información de una organización, para elevar el nivel de conocimientos que permitan un mejor apoyo a la toma de decisiones y desarrollo de acciones. Si se tuviera que resumir el principal objetivo de un sistema de información dentro de una organización, se podría afirmar que éste se encarga de entregar la información oportuna y precisa, con la presentación y formatos adecuados, a la persona que la necesita dentro de la organización para tomar una decisión o realizar alguna operación en el momento justo en que se necesite de disponer de dicha información (Gómez y Suarez, 2010). Además de apoyar la toma de decisiones, coordinación y control, los sistemas de información también pueden ayudar a los gerentes y trabajadores analizar problemas, visualizar temas complejos, y crear nuevos productos. Las tres actividades principales en un sistema de información que producen la información que las organizaciones necesitan para la toma de decisiones, operaciones de control, analizar problemas, y crear nuevos productos o servicios son: entrada, procesamiento, y la salida. Los sistemas de información también requieren de retroalimentación, que es la salida que se devuelve a los miembros apropiados de la organización para ayudarles a evaluar o corregir la etapa de entrada (Laudon y Laudon, 2011).

Information Retrieval o IR por sus siglas en inglés, hace referencia a recuperación de información, ésta área no es nueva, sino que se viene desarrollando desde finales de la década de los cincuentas. La recuperación de información solía ser una actividad que era de uso exclusivo de pocas personas como: bibliotecarios de referencia, asistentes legales, y similares buscadores profesionales. Ahora el mundo ha cambiado, y cientos de millones de personas se dedican a la recuperación de información todos los días cuando se utiliza un motor de búsqueda en la web o buscar en su correo electrónico, (Manning, Raghavan, y Schutze, 2009). "Los sistemas automatizados de recuperación de información se utilizan para reducir lo que se ha llamado "sobrecarga de información", la recuperación de la información puede verse vista de diferentes aspectos, de acuerdo a como el usuario interactúe.

Actualmente existen tres áreas que influyen en la recuperación XML (Fuhr et al, 2002): la primera son los lenguajes de consulta XML tradicional como XQuery (Boag et al, 2002, que proporcionan consultas complejas, pero sólo buscan coincidencias exactas. La segunda área está relacionada con los clásicos sistemas de información que utilizan bases de datos, estos han adoptado la posibilidad de almacenar datos semiestructurados (Fuhr et al, 2002) e incluyen el desarrollo de bases de datos con archivos XML. Por último la tercera área es la de recuperación de información (IR), los modelos clásicos de recuperación de información proporcionan clasificación por relevancia, pero no incluyen la estructura del documento, además, aplican un concepto de documento estático, por lo que las unidades de recuperación por lo general son documentos completos (Schlieder y Meuss, 2002).

\section{METODOLOGÍA}

Para el desarrollo del sistema de información presentado en este artículo se utilizó una estructura de proceso general para la ingeniería de software que consta de cinco actividades (Pressman, 2010), estas cinco actividades estructurales genéricas se usan durante el desarrollo de programas y fueron ejecutadas aplicando el modelo de la cascada, a veces llamado ciclo de vida clásico, que sugiere un enfoque sistemático y secuencial para el desarrollo del software. Con esta metodología se aprecian las 
características que permitieron desarrollar el sistema de información acorde a las necesidades de la pyme sujeto de estudio, soportado en una base de datos tradicional y una base de datos con archivos de texto usando recuperación XML para la búsqueda y extracción de información.

Fase 1 Comunicación: En esta fase se buscó entender los objetivos de los participantes respecto del proyecto, y reunir los requerimientos que ayuden a definir las características y funcionalidad del software. Las técnicas de recolección de información utilizadas fueron la observación directa y la entrevista. Las entrevistas fueron dirigidas hacia tres funcionarios relacionados con las actividades de gestión de los principales procesos que se realizan en la casa del embobinador asignados a las áreas de gerencia y servicios. Logrando identificar cada uno de los procesos relacionados con los servicios asociados a las órdenes de reparación, sus principales características, y se definieron los requerimientos que involucran el desarrollo del sistema de información para la pyme. Se pudo identificar en esta fase que los principales procesos son realizados en su mayoría de manera manual y en otros llevados a cabo, a través de hojas de cálculo que no brinda el nivel de seguridad de los datos ni características que pudieran ayudar a la efectividad y optimización de los procesos, ni a la mejora en la toma de decisiones.

Fase 2 Planeación: En esta fase se realizó un plan del proyecto de software - definiendo el trabajo de ingeniería de software al describir las tareas técnicas por realizar, los riesgos probables, los recursos que se requieren, los productos del trabajo que se obtendrán y una programación de las actividades. Se realizó un cronograma, identificando los hitos del proyecto y un presupuesto.

Fase 3 Modelado: En esta fase se crearon los diagramas utilizando UML como lenguaje para crear los diagramas que modelaran el sistema a fin de entender el panorama general -cómo se ve arquitectónicamente, cómo ajustan entre sí las partes constituyentes entre otras características.

Fase 4 Construcción: Esta fase combino la generación de código y las pruebas que se requieren para descubrir errores en éste. El sistema de información fue realizado como aplicación web, utilizando el patrón modelo vista controlador (MVC) (Leff y Rayfield, 2001), usando el lenguaje de programación Java Server Pages (JSP) (Fields et al, 2001) el cual permite crear páginas web dinámicas basadas en HTML. Para desplegar y correr el sistema de información se usó un servidor web compatible con contenedores servlet como Apache Tomcat.

Fase 5 Despliegue: El sistema de información fue entregado al personal de la pyme sujeto de estudio quien lo evalúo y brindó la retroalimentación necesaria.

\section{RESULTADOS Y DISCUSIÓN}

Aplicada la metodología se pudo elaborar el modelo del dominio del problema, mostrado en la figura 1. Elaborado el modelo del dominio se identificaron los siguientes requerimientos funcionales entre otros: registrar usuarios, dar de baja usuarios, iniciar y cerrar sesión, gestión de permisos de usuarios, gestionar las órdenes internas de trabajo (OIT), se debe permitir registrar las diferentes órdenes de trabajo que existen en la empresa debido a que dependiendo el tipo de motor así serán los datos registrados. Estas son: OIT motores AC monofásicos, OIT equipos y servicios varios, OIT generadores A.C, OIT motores y generadores D.C., OIT motores A.C. trifásicos. Nota: a cada OIT antes mencionada se le debe activar el formato de anexos si se requiere. Se debe permitir la modificación de las órdenes de trabajo mientras estas estén pre guardadas para ingresar datos posteriores hasta el momento del guardado final. La OIT finalizadas solo podrán ser modificadas por el usuario administrador. Otro de los requerimientos son buscar una OIT guardada: Se podrá buscar una OIT almacenada en el sistema para su visualización o modificación. Y por último y no menos importante generar reportes estadísticos de los indicadores definidos, para dar soporte a la toma de decisiones. Con el fin de atender los requerimientos funcionales expuestos anteriormente, se plantearon los siguientes casos de uso, figura 2. En la figura 2 se observa el diagrama de casos de uso, representa la descripción grafica de los requerimientos funcionales principales del sistema. Se pueden observar los usuarios involucrados y las funciones que cada uno cumple en el sistema.

La recuperación XML como técnica aplicada en este sistema de información, brinda herramientas prácticas y argumenta la eficiencia que se puede conseguir con la utilización de archivos XML en la búsqueda de información debido a su estructura organizada. El proceso llevado a cabo en esta aplicación consistió en revisar detalladamente el archivo XML asociado a un numero de una OIT, y aplicando un método de indexación selectiva, combinado con técnicas de indexación de archivos XML como: indexación clásica, indexación basada en elementos hojas e indexación distribuida, se deciden qué elementos son los que se indexen, descartando los restantes (Cacheda, Fernández \& Huete, 2011). En este caso puntual, cada OIT, tiene un diagrama a mano alzada del motor al cual se le va a realizar el trabajo, ya sea de mantenimiento o reparación. 


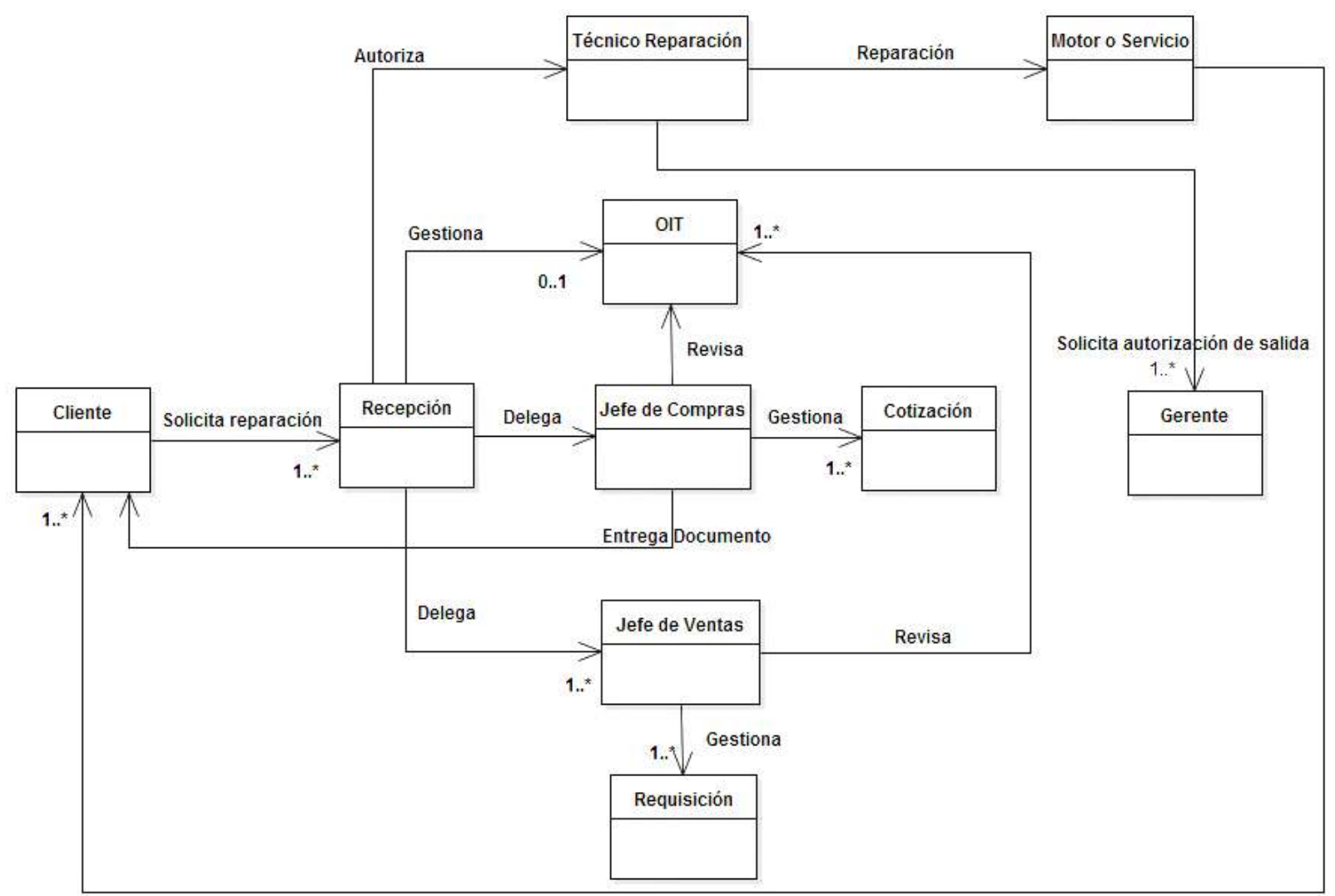

Fig. 1: Modelo del dominio

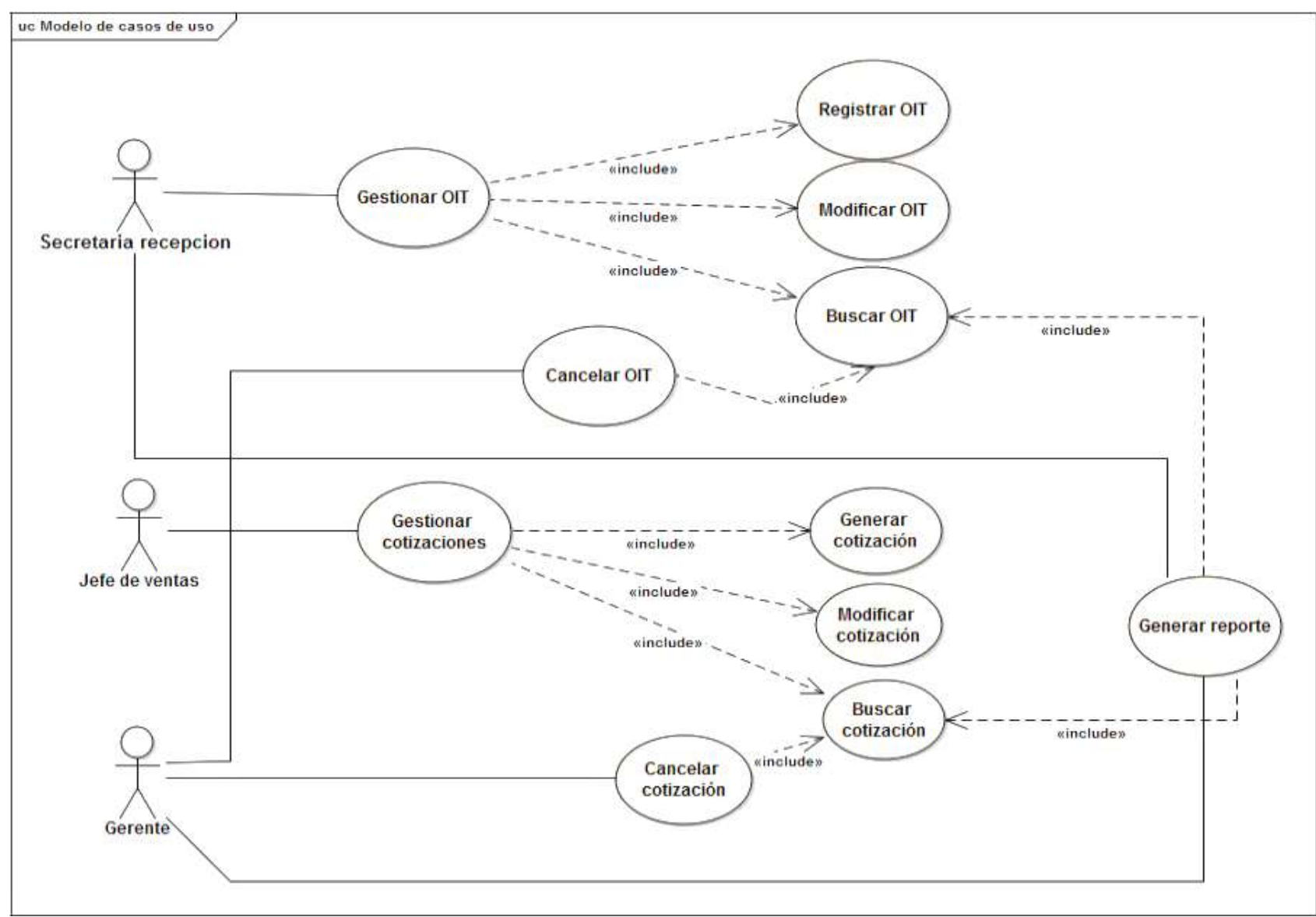

Fig. 2: Diagrama de casos de uso 
Este diagrama es una representación útil tanto para el operador como para la gestión de trabajos futuros asociados a ese motor. El sistema de información almacena para cada OIT el diagrama realizado a cada motor en forma de imagen y por cada imagen en cuestión genera un archivo XML con los metadatos del diagrama.

El sistema de información utiliza una bases de datos clásica, con la posibilidad de almacenar datos semiestructurados (Fuhr et al., 2002) para los datos textuales de cada OIT e incluyen el desarrollo de bases de datos con archivos XML para los diagramas del motor o motores asociados a la OIT. Las consultas que permite realizar el sistema son consultas clásicas de solo texto y buscar incluso por el diagrama realizado al motor, se procesa revisando coincidencias textuales y asignando pesos a estas coincidencias ordenando según su grado de relevancia, dándole más prioridad a la OIT asociada que contenga los elementos más apropiados. La figura 3 , muestra el proceso de consulta cuando se realiza directamente a los datos almacenados en archivos XML.

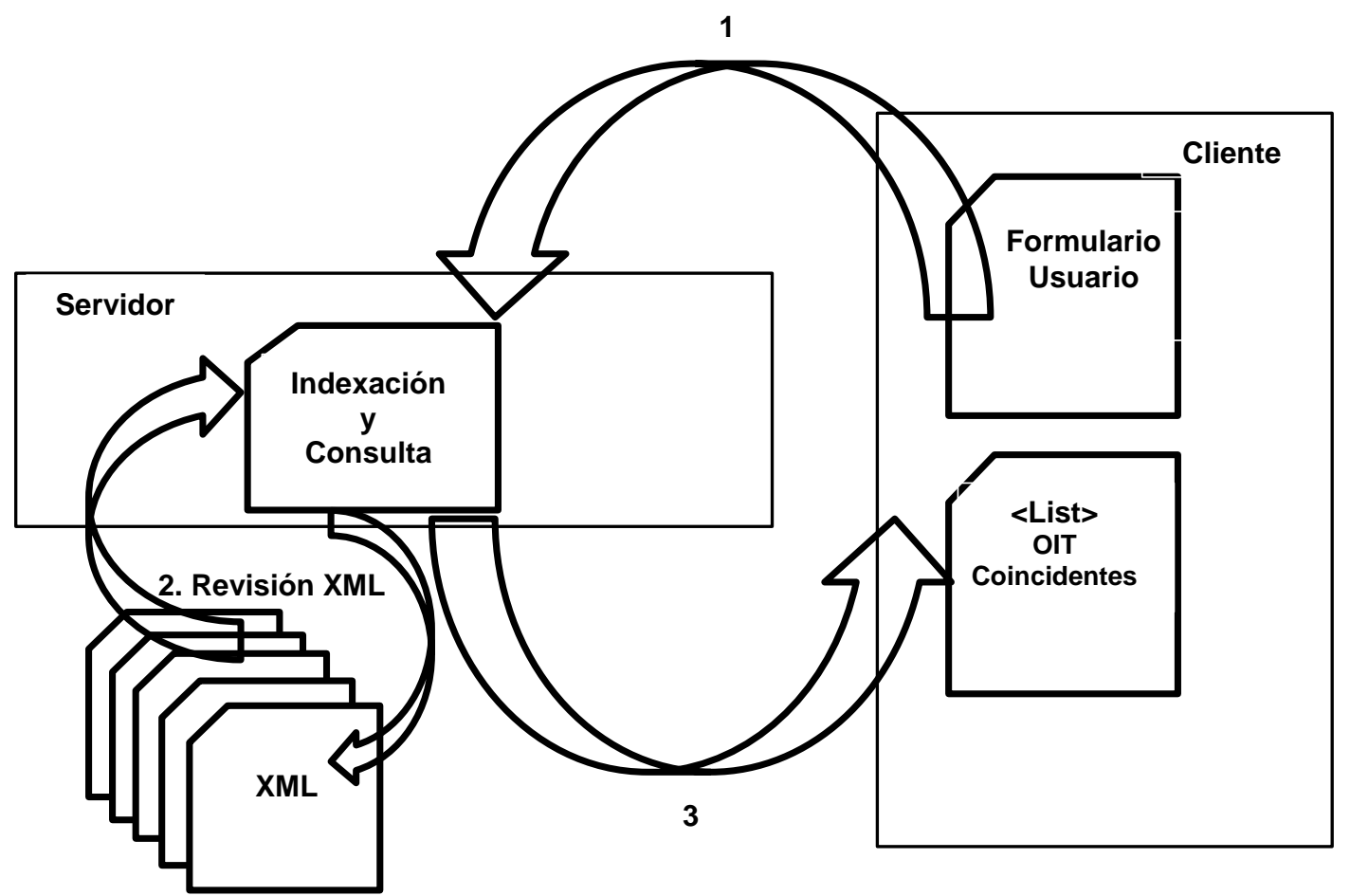

Fig. 3: Representación esquemática del proceso de búsqueda de una OIT basado en recuperación XML

Se realizó una prueba de usabilidad al sistema realizada por parte de los directos involucrados en la ejecución de los procesos. Nielsen define la usabilidad, como la facilidad de uso, más específicamente, hace referencia a la rapidez con que se puede aprender a utilizar algo, la eficiencia al utilizarlo, cuál es su grado de propensión al error, y cuanto le gusta lo evaluado a los usuarios (Nielsen, 1994). La prueba de usabilidad realizada, estuvo relacionada con la facilidad de los empleados de la casa del embobinador para usar el sistema de información, gestionar las OIT y realizar búsquedas relacionadas con estas. En dicha prueba en escala de 1 a 5 , donde 1 es pobre y 5 es excelente, se le pedía al usuario final que evaluara el sistema de información de acuerdo a cinco atributos (Nielsen, 1994): El primer atributo es facilidad de aprendizaje: Indica qué tan fácil es aprender la funcionalidad básica del sistema, como para ser capaz de realizar precisamente las tareas que desea realizar el usuario. Eficiencia: El segundo atributo es la eficiencia se determina por el número de transacciones por unidad de tiempo que el usuario puede realizar usando el sistema. Lo que se busca es la máxima velocidad de realización de tareas del usuario. Cuanto mayor es la eficiencia de un sistema, más rápido es el usuario al utilizarlo, y el trabajo se realiza con mayor rapidez. El tercero es la presentación visual apropiada:

El concepto de sistema se materializa al realizar el diseño de la parte visual de la interacción, es decir, la "interfaz gráfica de usuario". Hay una serie de normas provenientes del campo del diseño gráfico sobre cómo escoger los colores, tipos de letra, la disposición de los elementos en una ventana, etc. El cuarto es manejo de errores: Indica cómo el sistema puede manejar los errores que el usuario comente mientras se encuentra operándolo. Resulta importante que el sistema pueda notar inmediatamente las acciones erróneas y después así deshacerlas. Y el quinto es satisfacción: Éste es el atributo más subjetivo. Indica la impresión subjetiva que el operador del sistema obtiene del mismo. Para ello se utilizó cuestionarios, encuestas, entrevistas. 
La encuesta de usabilidad fue realizada por tres personas: el asistente de producción, subgerente y gerente, en esta encuesta se alcanzan valores máximos obtenidos en la prueba, en el atributo de facilidad de aprendizaje el cual se califica con el número 5 por todos los sujetos, al menos uno de los sujetos encuestados calificó con 5 los atributos de satisfacción, manejo de errores, presentación visual apropiada y satisfacción.

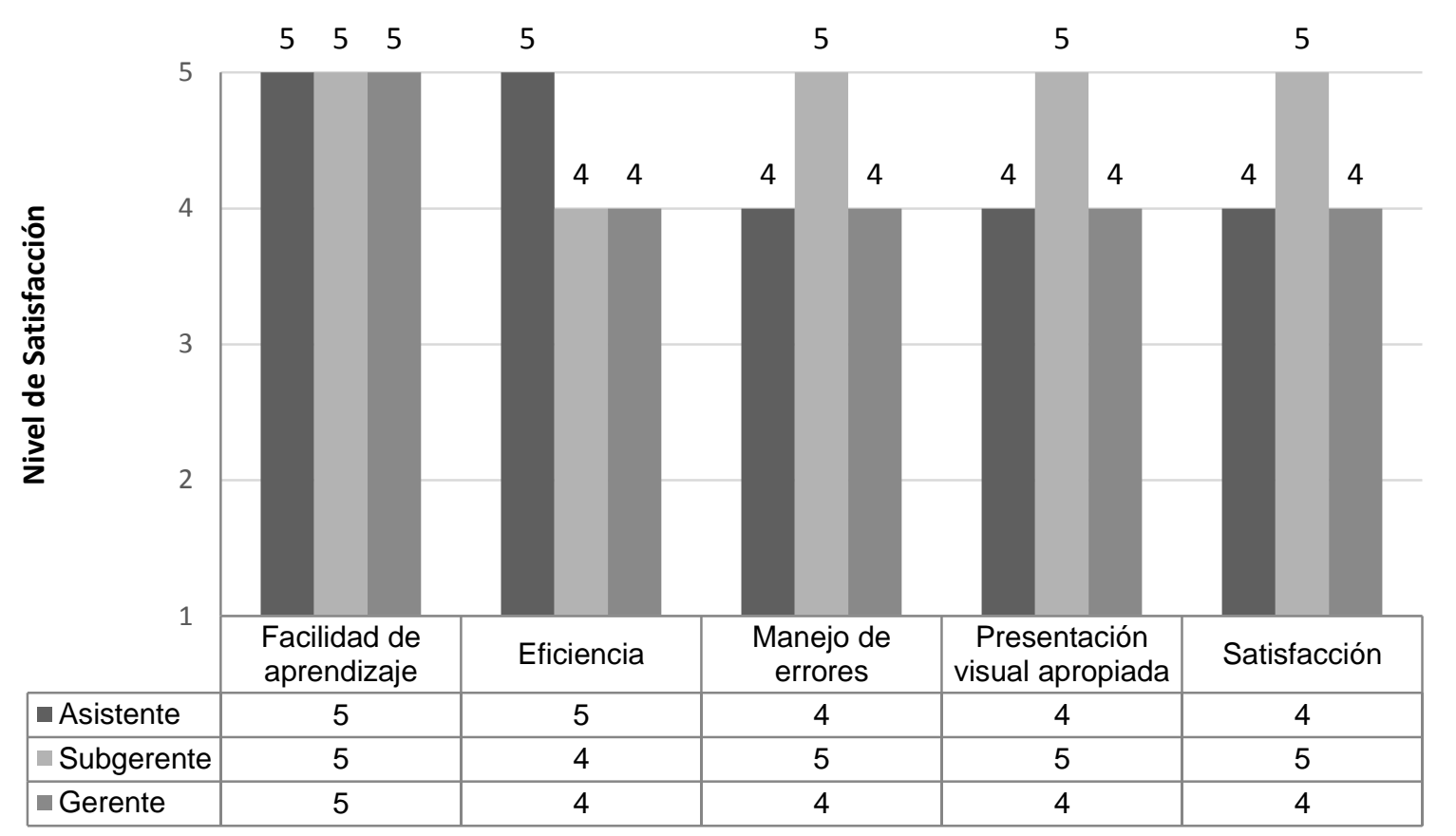

Fig. 4: Resultados por sujeto evaluado de la prueba de usabilidad

La implantación de sistemas de información de este tipo, se convierte en un reto no solo para las pymes del sector metalmecánico, si no para todas en general, puesto que la mayoría de estas son resistentes al cambio, sumado al hecho que para poder usar estas herramientas se hace necesario que las pymes asuman los costos de adquisición de la infraestructura tecnológica de hardware y software necesaria para que el sistema de información funcione correctamente. Adicional la contratación de personal humano calificado para el uso, administración y posterior mantenimiento de todo lo que tiene que ver con hardware y software, convirtiéndose en un desafío importantísimo que las pymes no pueden hacer un lado si quieren adquirir ventajas competitivas que les permitan mantenerse en el mercado actual. Por último y no menos importante, la organización interna de la pyme, el conocimiento de sus procesos de negocios e incluso la certificación de sus procesos, permiten que la implantación de este tipo de sistemas sea más fácil para aquellas pymes que cumplan con estos aspectos, como es el caso de la empresa usada para probar la herramienta, pues esta contaba con un sistema de gestión de calidad certificado bajo la normatividad NTCISO 9001:2008, lo que facilita grandemente el uso y apropiación del sistema.

Se recomienda para una segunda versión del sistema de información, ampliar la funcionalidad del sistema enfocado al seguimiento del proceso de gestión de la OIT y la obtención de reportes con información estadística y gráficos que permitan describir en forma visual el desempeño de la empresa y sus departamentos en el manejo de los procesos relacionados con las OIT. También se podría considerar la posibilidad de incluir una aplicación móvil como un módulo adicional que permita ingresar la información sobre la OIT por el mismo técnico desde el taller de reparación y se disminuya el uso de formatos en papel.

\section{CONCLUSIONES}

De los resultados y el análisis presentado se pueden obtener las siguientes conclusiones sobre el enfoque propuesto y la herramienta que lo soporta: 1) La gestión de OIT en la casa del embobinador cuenta con sistema de información que soporte la dinámica del proceso y le da un valor agregado que repercute en ventajas competitivas para la empresa. 2) La aplicación de recuperación XML, agrega ventajas y características de IR que permite el manejo de los datos, documentos, formularios y que mantener la integridad del archivo histórico así como la mejora en las búsquedas de información. 3) Apoya la toma de decisiones en el manejo de reparaciones basadas en órdenes históricas. 4) Permite ahorrar costos y recursos físicos para el manejo del archivo. 5) Cualquier proceso de innovación tecnológica tiene muchos 
beneficios proyectados a corto, mediano y largo plazo, que al materializarse a través del uso este tipo de sistemas en las organizaciones permiten que los procesos cotidianos generen sinergia en la compañía, es decir, se aumente los niveles de integración y se tomen decisiones acertadas y ajustadas a la realidad.

\section{REFERENCIAS}

BID - Banco Interamericano de Desarrollo, Desencadenar el crédito: Cómo ampliar y estabilizar la banca, Informe Progreso Económico y Social en América Latina (2005), http://www.iadb.org. Acceso: 9 de Julio (2014).

Bray, T. y otros cuatro autores, Extensible markup language (XML). World Wide Web Consortium Recommendation REC-xml-19980210 (1998), http://www.w3.org/TR/1998/REC-xml-19980210. Acceso: 6 de Julio (2014).

Cacheda, F., Fernández, J. M. y J. F. Huete, Recuperación de información: un enfoque práctico y multidisciplinar, Editorial Ra-Ma, España (2011).

Carrillo, M. H., y Franky, C. Modelo SCLOUDPY para la Gestión de Pedidos en la Nube, doi: 10.4067/S0718-07642014000400006, Inf. Tecnol. (en línea), 25(4), 35-42, (2014).

El Congreso de la República de Colombia, Ley 590 de 2000, Por la cual se dictan disposiciones para promover el desarrollo de las micro, pequeñas y medianas empresa. Diario Oficial No. 44.078 de 12 de julio (2000).

Fuhr, N. y otros tres autores. INEX: Initiative for the Evaluation of XML retrieval. In Proceedings of the SIGIR 2002 Workshop on XML and Information Retrieval, Vol. 2006, pp. 1-9 (2002).

Fields, D. K., Kolb, M. A. y S. Bayern, Web Development with Java Server Pages. Manning Publications Co, (2001).

Galindo, A. G., El tamaño empresarial como factor de diversidad. Edición electrónica Grupo EUMED. Universidad de Málaga (2005), http://www.eumed.net/ libros/2005/agl3/ Acceso: 28 mayo (2014).

Gómez, A. y C. Suárez, Sistemas de información: herramientas prácticas para la gestión. México: Alfaomega Ra-Ma Grupo editor. Pág. 34-35 (2010).

Hassler, V. Open source libraries for information retrieval, doi: 10.1109/MS.2005.131, Software, IEEE: 22(5), 78-82 (2005).

Hernández, A., Marulanda, C.E., y López, M. Análisis de Capacidades de Gestión del Conocimiento para la Competitividad de PYMES en Colombia, doi: 10.4067/S0718-07642014000200013, Inf. Tecnol. (en línea), 25(2), 111-122 (2014).

Laudon, K. C., y J. P. Laudon, Essentials of management information systems. Upper Saddle River: Editorial Pearson, (2011).

Leff, A. y J. T. Rayfield, Web-application development using the model/view/controller design pattern, doi: 10.1109/EDOC.2001.95042, In Enterprise Distributed Object Computing Conference, 2001. EDOC'01. Proceedings. Fifth IEEE International (pp. 118-127). IEEE, (2001).

Luk, R. W. y otros cinco autores. A survey in indexing and searching XML documents. Journal of the American Society for Information Science and Technology: 53(6), 415-437 (2002).

Martínez, F. J., Recuperación de información: modelos, sistemas y evaluación. Murcia, Kiosko, (2004).

Manning, C. D., Raghavan, P. y H. Schütze, Introduction to information retrieval (On line edition), Cambridge, Cambridge University Press, (2009).

Malhotra, M. y otros cinco autores, Expanding Access to Finance: Good Practices and Policies for Micro, Small, and Medium Enterprises. USA: World Bank Institute, (2007).

Nielsen, J. Usability engineering. Elsevier. Chapter 2.2, p. 26, (1994). 
Pokorný, J., Web searching and information retrieval, doi: 10.1109/MCSE.2004.24. Computing in Science \& Engineering: 6(4), 43-48 (2004).

Pressman, R. S., Ingeniería del Software Un enfoque práctico. 7ª edición, Editorial McGraw Hill, (2010).

Puello, P., Cabarcas, A., y Martelo, R.J. Sistema de Información Gerencial para la Administración de Recursos Educativos, doi: 10.4067/S0718-50062013000500003, Formación universitaria. (en línea), 6(5), 13-20, (2013).

Rodríguez, A. Z. y otros seis autores, Plan estratégico y prospectivo de innovación y desarrollo científico y tecnológico del departamento de Bolívar. Cámara de Comercio de Cartagena. (2011). http://www.cccartagena.org.co/descargar archivo.php?f=20120509111338 Plan Estrategico DTCB.pdf. Acceso: 16 de agosto (2013).

Schlieder, T. y H. Meuss, Querying and ranking XML documents, doi: 10.1002/asi.10060, J. Am. Soc. Inf. Sci. Techn. (en línea), 53(6), 489-503 (2002).

United Nations, Information economy report: The Software Industry and Developing Countries, United Nations Conference on trade and development, United Nations Publications, (2012).

Vélez, B., Cabarcas, A. y L. Malavé, TIN: an interactive image navigator providing ubiquitous access to distributed geo-spatial data, doi: 10.1109/ITCC.2004.1286476, Proceedings. ITCC 2004. International Conference on, 1, 337-343. IEEE, (2004).

Winter, J. y O. Drobnik, An architecture for XML information retrieval in a peer-to-peer environment, doi: 10.1145/1316874.1316878. In Proceedings of the ACM first Ph. D. workshop in CIKM, 17-24, ACM, (2007).

Zevallos, E., Restricciones del entorno a la competitividad empresarial de América Latina, Fundes, 151 (2007). 
